\title{
Clinical and surgical assistance in prostate cancer during the COVID-19 Pandemic: implementation of assistance protocols
}

Lara Rodriguez Sanchez ${ }^{1,2}$, Xavier Cathelineau ${ }^{1}$, Alexis M. Alva Pinto ${ }^{3}$, Ángel Borque-Fernando ${ }^{2,4}$, Maria Jesús Gil ${ }^{2,4}$, Chi-Hang Yee ${ }^{5}$, Rafael Sanchez-Salas ${ }^{1}$

${ }^{1}$ Department of Urology, L'Institut Mutualiste Montsouris, Université Paris-Descartes, Paris, France; ${ }^{2}$ Department of Urology, Hospital Universitario Miguel Servet, Zaragoza, Spain; ${ }^{3}$ Department of Urology, Clinica Oncosalud, Lima, Peru; ${ }^{4}$ IIS-Aragon, Zaragoza, Spain; ${ }^{5}$ Department of Surgery, S.H. Ho Urology Centre,The Chinese University of Hong Kong, Hong Kong

\section{ABSTRACT}

Purpose: Propose an approach of prostate cancer (PCa) patients during COVID-19 pandemic. Material and Methods: We conducted a review of current literature related to surgical and clinical management of patients during COVID-19 crisis paying special attention to oncological ones and especially those suffering from PCa. Based on these publications and current urological guidelines, a manual to manage PCa patients is suggested.

Results: Patients suffering from cancer are likely to develop serious complications from COVID-19 disease together with an increased risk of postoperative morbidity and mortality. Therefore, the management of oncological patients should be taken into special consideration and most of the treatments postponed.

In case the procedure is not deferrable, it should be adapted to the current situation. While the shortest radiotherapy (RT) regimens should be applied, surgical procedures must undergo the following recommendations proposed by main surgical associations.

PCa prognosis is generally favourable and therefore one can safely delay most of the biopsies up to 6 months without interfering with survival outcomes in the vast majority of cases. In the same way, most of the localised PCa patients are suitable for active surveillance (AS) or hormonal therapy until local definitive treatment could be reconsidered. In metastatic as well as castration resistant PCa stages, adding androgen receptor targeted agents (abiraterone, apalutamide, darolutamide or enzalutamide) to androgen-deprivation therapy (ADT) could be considered in high risk patients. On the contrary, chemotherapy, immunotherapy and Radium-223 must be avoided with regard to the consequence of hematologic toxicity and risk of COVID-19 infection because of immunodepression.

Conclusions: Most of the biopsies should be delayed while AS is advised in those patients with low risk PCa. ADT allows us to defer definitive local treatment in many cases of intermediate and high risk PCa. In regard to metastatic and castration resistant PCa, combination therapies with abiraterone, apalutamide, darolutamide or enzalutamide could be considered. Chemotherapy, Radium-223 and immunotherapy are discouraged.

\section{ARTICLE INFO}

Lara Rodríguez Sánchez

http://orcid.org/0000-0001-6939-2645

Keywords:

Prostate cancer, familial

[Supplementary Concept];

GRADE Approach; COVID-19

[Supplementary Concept];

Pandemics

Int Braz J Urol. 2020; 46 (Suppl 1): 50-61

Submitted for publication:

April 25, 2020

Accepted after revision:

May 10, 2020

Published as Ahead of Print: June 05, 2020 


\section{INTRODUCTION}

They are called Guidelines, not God's lines

The outbreak of coronavirus that cause the disease COVID-19 has not only created a pandemic situation and a global crisis but beyond imagination, it has completely modified our way to look at medical information and its clinical application.

Medical Guidelines, without a doubt, are of utmost importance and a great deal of work is continuously deployed to offer our patients the highest level of patient care. Every society solidly invests on the training of the young generation to develop novel ideas, then exposing those ideas to a scientific method, eventually obtaining evidence and more importantly reaching a high level of recommendation. Simultaneously, our younger peers are actively taught to verify this information in detail, selecting the best of it and to create a number of standardized practices aiming to objectively guide therapeutic options. Particularly in the case of oncology there are so many exceptions that do not necessarily fit the typical case for one option or another (1). In these occasions tumor boards and faculty discussions may provide a rational and adoptable treatment option.

Nowadays days we face an unfamiliar enemy, SARS-CoV-2, an RNA virus with low mutational process but a high recombination potential allowing it to switch hosts in a rather timely fashion (2). Nevertheless, whereas we hold much basic knowledge on the anatomy of this new type of coronavirus which is able to cause severe respiratory illness in $20 \%$ of patients (5\% of them requiring ventilation and intensive care) (3), no high level evidence recommendations are available to deal with the challenge it has created to humanity.

Today, we had no choice but to look back at " experience medicine " and use the creator of Sherlock Holmes, Sir Arthur Conan Doyle, approach to identify and solve problems. What do we have in our medical armamentarium that could deal with this threat? Researches around the World are dealing with this question, and of utmost importance, we must understand that during this pandemic, cancer does not stop and some specific patients still need priority treatment.

Our aim is to propose an approach for prostate cancer $(\mathrm{PCa})$ patients management during COVID-19 pandemic.

\section{MATERIAL AND METHODS}

We reviewed most of the ongoing recommendations given by the main health, surgical and urological associations around the World, such as the World Health Organization (WHO), the National Comprehensive Cancer Network (NCCN), the European Association of Urology (EAU) and the British Association of Urological Surgeons (36). In addition, publications related to COVID-19 pandemic were also reviewed putting special interest in those focused on surgical management of patients, as well as cancer, in particularly PCa.

Afterwards, the authors propose a practical guide to manage PCa during COVID-19 outbreak based on current urological guidelines. Such proposal is adapted to the special condition we face today.

\section{RESULTS}

Patient selection to perform a PCa Biopsy and Conditions

It has been reported that SARS-CoV-2 is present in the stool of COVID-19 patients and fecal-oral transmission is possible. While it has not been demonstrated that the prostate biopsy procedure itself would be a way of COVID-19 transmission, we advise to avoid or defer almost all prostate biopsies (7-9).

\section{Whom to biopsy}

In cases where risk factors for high risk $\mathrm{PCa}$ are present -prostate specific antigen (PSA) $>20$, PSA doubling time (PSA-DT) $<6$ months, suspicious of clinical T3 disease, and/or local or systemic symptoms-, biopsy can be delayed up to 3 months. On the other hand, in the absence of high risk factors, biopsy may be postponed till 3 to 6 months later $(6,8)$, or even 12 months according to NCCN recommendations (7) (Table-1). 
Table 1 - Management of clinical suspicion of PCa and localized PCa during the COVID-19 era.

\begin{tabular}{lcc}
\hline Tumor stage & Recommendations & Comments \\
\hline $\begin{array}{l}\text { Clinical suspicion (elevated } \\
\text { PSA and/or abnormal DRE) }\end{array}$ & 1. Presence of High risk PCa factors & PCa prognosis generally \\
& - PSA-DT doubling time $<6$ months & favourable can safely delay \\
most biopsies up to 6 to 12 \\
months without interfering \\
with survival outcomes in the \\
vast majority of cases (6-8).
\end{tabular}

2. Absence of High risk PCa factors

Biopsy may be postponed 3 to 6 months (8).

\section{Localized very low, low and risk favorable intermediate-risk diseases.}

\section{Localized unfavorable} intermediate risk
AS must be prioritized while RP as well as RT should be deferred.

Delay local definitive treatment

Start ADT 6-monthly formulations

Prioritize definitive treatment if it is available

Localized High-risk and very high-risk
Start ADT 6-monthly formulations

Consider ADT followed by RT in selected patients

Consider ADT followed by RP in selected patients
NCCN and EAU PCa guidelines currently recommend AS (15,

16).

Delay RP and RT may not imply a very high impact on oncological outcomes (13,

14).

NCCN and EAU PCa guidelines currently recommend short course of ADT added to RT

$(15,16)$.

Preoperative ADT studies show a lack of benefit in prolonging overall survival but an improvement in pathological variables (17).

Initiation of ADT must be the standard of care in these patients until local therapy could be reconsidered as the coronavirus crisis improves or ends.

The benefit of neoadjuvant ADT has already been widely verified before RT (17).

Preoperative ADT studies show a lack of benefit in prolonging overall survival but an improvement in pathological variables (17). 
In regard to Multi-Parametric Magnetic Resonance Imaging (mpMRI), the EAU recommends upfront pre-biopsy mpMRI if resources allow. However, if the patient is suspected to be liable to risk of progression and metastasis, biopsy can be performed without prior MRI (6).

Considering a possible fecal-oral COVID-19 transmission no rectal lavage for preparation and complete protective personal equipment (PPE) during procedure are advisable. Negative-pressure rooms should be utilized when possible (6-9).

\section{Patient Approach}

In those patients with localized $\mathrm{PCa}$, the two available options to treat them with curative intent, namely radiotherapy (RT) and radical prostatectomy (RP), must be both postponed as much as possible during coronavirus crisis (10, 11). In the case of metastatic PCa patients the use of some systemic treatments may be compromised as a consequence of an increase in the number of visits to health centers and the risk of iatrogenic infection that it would entail.

Nowadays the main question to be resolved is how long our patients can wait for a treatment without interfering with oncological outcomes. While this doubt is clarified we propose the following management.

\section{Localized disease}

Very low, low and risk favorable intermediate-risk diseases

A recent prospective, open-enrollment cohort study showed a risk of cancer death or metastasis lower than 1\% over $15 y$ y follow-up in Grade Group 1 PCa patients who underwent Active surveillance (AS) (12). According to this study as well as PROTECT and PIVOT trials $(13,14)$, NCCN and European PCa guidelines currently recommend or propose AS as a good management option in this group of patients having favorable outcomes $(15,16)$. Therefore, AS must be prioritized while RP as well as RT should be deferred until restrictions to contain the spread of COVID-19 are over (Table-1).

Follow-up biopsies and PSA-tests should be postponed by $>3$ months from the preplan- ned appointment in order to decrease the number of visits to hospital and to promote social distancing (6).

\section{Unfavorable intermediate risk}

Despite the fact that AS for these stages of the disease is not contemplated in the current guidelines $(15,16)$, given the extraordinary situation in which we find ourselves in, RP and RT should be postponed with a believably not very high impact on specific cancer mortality $(13,14)$.

Short course (4-6 months) of androgen-deprivation therapy (ADT) added to RT are indicated in these patients $(15,16)$ while ADT prior to RP might be considered during COVID 19 pandemic. Although it is well known that this last approach is not associated with survival benefits it is also related to better pathological results (17). For these reasons, we recommend the initiation of androgen blockage.

In relation to the possible effect that a prolonged neoadjuvant treatment may have on oncological outcomes it has been observed that extending neoadjuvant ADT therapy duration prior to RT from 8 to 28 weeks neither significantly improve nor worsen oncological outcomes on patients with unfavourable intermediate risk PCa (18). These results suggest that we may safely delay the need to definitive local treatment for 4-6 months $(9,18,19)$ (Table-1).

Keeping into consideration that those patients with a PCa grade group 3 could have an increased risk for eventual metastases (19) as well as a five-fold increased risk of PCa mortality compared to grade group 2 (20), we suggest to continue follow-up PSA-tests every 3 months and provide the results by telehealth.

\section{High-risk and very high-risk}

Initiation of ADT must be the standard of care in these patients until local therapy could be reconsidered as the coronavirus crisis improves or ends. The benefit of neoadjuvant ADT has already been widely verified before RT (17).

In view of a lack of benefit in prolonging overall survival, current urological gui- 
delines strongly discourage the use of elective neo-adjuvant ADT in patients who are going to undergo RP outside of clinical trials (15-17). However, preoperative ADT studies have shown a significant reduction in positive surgical margins and downstaging along with an improvement in other pathological variables such as lymph node involvement. Additionally, these results tended to be better if neo-adjuvant ADT was prolonged from 3 to 6 or 8 months prior to surgery (17).

Intensive androgen blockage prior to RP is currently under study with favorable preliminary results, but further study is necessary (21).

In case of detecting patients with a rapid PSA-DT ( $\leq 3$ months) timely therapy could be indicated and the benefits of immediate treatment must be weighed against the risk associated to iatrogenic exposure to COVID-19 (11).

As long as there is a limited availability of operating rooms, material and human surgical resources which make it impossible to perform RP, RT could be an alternative. Within RADS (Remote visits, Avoidance, Deferments, and Shortening of radiotherapy) framework created by Radiation Oncologist, shortening of the RT treatment is the fundamental principle for these high risk patients without compromising the oncological outcomes $(4,11)$ (Table-1).
Like what was proposed previously for patients with unfavourable intermediate-risk PCa, we suggest to maintain quarterly PSA monitoring.

Unfavourable features after radical prostatectomy According to preliminary results from ARTISTIC meta-analysis presented at ESMO 2019 Congress, event-free survival is not improved with adjuvant radiotherapy (ART) compared to salvage radiotherapy (SRT) in patients with combined high-risk features (pT3-T4/R1/ GS (8-10, 22). Despite ART remains the recommended treatment option until more evidence to suggest otherwise $(15,16)$, we strongly advise SRT as a safe alternative (Table-2).

\section{Biochemical recurrence (BCR)}

The real impact of BCR in cancer mortality is currently unknown whereas recent studies suggest that just a subgroup of patients would develop progressive disease following $\mathrm{BCR}$ after RP with less optimistic results in case of RT failure. In this sense, patients may be stratified into EAU Low-Risk or High-Risk BCR according to PSA-DT, pathological ISUP grade and interval to biochemical failure (23).

According to the above, in case of clinical suspicion of BCR the authors suggest to postpone

Table 2- Management of unfavourable features after radical prostatectomy or biochemical recurrence after local treatment of PCa during the COVID-19 era.

\begin{tabular}{lcc}
\hline Tumor Stage & Recommendations & Comments \\
\hline $\begin{array}{l}\text { Unfavourable features after radical } \\
\text { prostatectomy }\end{array}$ & Avoid adjuvant RT. & According to ARTISTIC meta- \\
& & analysis, event-free survival \\
& is not improved with ART \\
& compared to SRT in patients \\
& with combined high-risk \\
& features (pT3-T4/R1/GS \\
& $8-10)(22)$.
\end{tabular}

\section{Biochemical recurrence}

\begin{abstract}
Delay complementary studies as well as salvage treatments, especially in EAU low-risk cases.
\end{abstract}

Offer salvage treatment for those patients with highrisk $B C R$ if it is available. If not, neoadjuvant $A D T$ could be considered (6).
Recent studies suggest that just a subgroups of patients would develop progressive disease following BCR after local treatment (23).

$\mathbf{R T}=$ Radiotherapy; $\mathbf{A R T}=$ Adjuvant Radiotherapy; $\mathbf{S R T}=$ Salvage Radiotherapy; $\mathbf{E A U}=$ European Association of Urology $\mathbf{B C R}=$ Biochemical Recurrence; $\mathbf{A D T}=$ Androgen deprivation therapy 
complementary studies as well as salvage treatments during COVID-19 pandemic, especially in Low-Risk cases. On the other hand, we propose to offer salvage treatment for those patients with High-Risk BCR if it is available. If not, neoadjuvant ADT could be considered (6) (Table-2).

Non metastatic castration- resistant prostate cancer

Three randomised phase III trials, PROSPER, SPARTAN and ARAMIS showed a significant metastatic free survival benefit in non-metastatic castration- resistant PCa patients treated with enzalutamide vs. placebo, apalutamide vs. placebo or darolutamide vs. placebo, respectively. Therefore, current guidelines strongly recommend these drugs to patients with castration- resistant $\mathrm{PCa}$, absence of metastases and PSA-DT $<10$ months. Taking into account that survival benefit was not proven after 20 months of follow-up as well as potential adverse events, we recommend these drugs in high selected patients during the $\mathrm{CO}$ VID-19 pandemic $(1,15,16)$ (Table-3).

Metastatic disease

Metastatic castration- sensitive prostate cancer

ADT must be initiated according to current standard of care $(15,16)$, with the six-month formulations being the best choice $(4,5)$.

In regard to Intermittent $\mathrm{ADT}$, it requires a closer PSA and testosterone monitoring in addition to possible images so it should be avoided in order to minimize hospital attendance.

In the last few years combination castration therapy with the new hormonal treatments (abiraterone, apalutamide or enzalutamide) has demonstrated benefits in terms of survival com- pared to ADT alone. Abiraterone acetate and prednisone or apalutamide added to ADT significantly reduce the risk of death by an amount equal to 28 and $33 \%$ respectively $(24,25)$ while enzalutamide plus ADT reduces radiographic progression-free survival or deaths by $60 \%$ (26).

The median age of patients who are candidates for combination hormonal treatments is around 70 (24-26). Although age is a potential risk factor for mortality of adult inpatients with COVID-19 (27) and these new drugs imply a closer follow-up, agreeing with the EAU, we suggest to offer immediate systemic treatment within $<6$ moths as long as a correct follow-up by telemedicine can be guaranteed (6).

In case the use of combined hormonal treatment is contemplated, we suggest to avoid abiraterone since the use of corticosteroids in population infected with SARS-CoV-2 is not yet completely clarified $(5,28)$.

With respect to chemotherapy, it must be avoided as much as possible being replaced by ADT or ADT in combination with androgen receptor targeted agents in order to reduce the number of clinical visits and haematological toxicity without compromising oncological outcomes (Table-4).

\section{Metastatic castration- resistant prostate cancer}

For castration-resistant metastatic patients, ADT must be maintained.

Abiraterone significantly improves overall survival among patients who previously receive chemotherapy compared to ADT alone. Nevertheless, improvement in median survival is not more than 5 months (29). In those patients who have not received chemotherapy, median overall survival is also improved from 30,3 to 34,7 months

Table 3 - Management of non metastatic castration- resistant PCa during the COVID-19 era.

\begin{tabular}{lcc}
\hline Tumor Stage & Recommendations & Comments \\
\hline $\begin{array}{l}\text { Nonmetastatic castration- resistant } \\
\text { prostate cancer }\end{array}$ & $\begin{array}{c}\text { Consider combination castration therapy with the new } \\
\text { hormonal treatments (apalutamide, darolutamide, } \\
\text { enzalutamide) in high selected patients. }\end{array}$ & $\begin{array}{c}\text { These drugs have } \\
\text { demonstrated benefits in } \\
\text { terms of metastatic free } \\
\text { survival in patients with PSA- } \\
\text { DT }<10 \text { months }(1,15,16) .\end{array}$ \\
& & ind
\end{tabular}

PSA-DT = Prostate Specific Antigen - Doubling Time 
Table 4 - Management of metastatic PCa during the COVID-19 era.

\begin{tabular}{lll}
\hline Tumor stage & Recommendations & Comments \\
\hline
\end{tabular}

Metastatic castrationsensitive prostate cancer
ADT 6-months formulations must be initiated. Avoid intermittent ADT.

Consider combination castration therapy with the new hormonal treatments (abiraterone, apalutamide or enzalutamide).

Prefer apalutamide or enzalutamide to abiraterone.

Avoid CTx.

Metastatic castrationresistant prostate cancer
ADT 6-months formulations must be maintained.

Consider combination castration therapy with the new hormonal treatments (abiraterone, enzalutamide).

Prefer enzalutamide to abiraterone.

Avoid CTx.

Avoid Immunotherapy (Sipuleucel-T).

Avoid Radium-223.

Avoid starting denosumab or zoledronic acid.

In those patients under treatment, denosumab may be maintained while zoledronic acid should be delayed.
ADT is the current standard of care $(15,16)$.

Intermittent ADT requires a closer PSA and testosterone monitoring in addition to possible images.

These drugs have demonstrated benefits in terms of survival compared to ADT alone (24-26).

Effect of corticosteroids in population infected with SARS-CoV-2 is not yet clear $(5,28)$.

CTx is associated with hematological toxicity and implies multiple visits to the hospital (6).

ADT maintenance is the current standard of care $(15,16)$.

These drugs have demonstrated benefits in terms of survival compared to ADT alone (29, 30-32).

Effect of corticosteroids in population infected with SARS-CoV-2 is not yet clear $(5,27)$.

CTx is associated with hematological toxicity and implies multiple visits to the hospital (6).

Sipuleucel-T might cause cytokine release while cytokines as IL-6 have been directly related to the most aggressive forms of COVID-19 $(4,34)$.

Radium-223 is associated with overall survival benefit by 3,6 (in the absence of visceral metastases) compared to ADT alone, but it is also associated to hematologic toxicity (35).

Denosumab or zoledronic acid have no impact on overall survival but could generate osteonecrosis of the jaw or hypocalcaemia $(36,37)$.

Denosumab can be administrated in its monthly subcutaneous formulation while zoledronic acid requires monthly hospital intravenous administration. 
(30). Similar results are observed with enzalutamide plus ADT. It has been reported an improvement in median overall survival of 5 months in those patients already treated with chemotherapy(31) and 2 months in chemo-naïve patients (32).

In case we decide to introduce abiraterone or enzalutamide, we must choose enzalutamide for the reasons previously mentioned $(5,27)$.

Chemotherapy also increases median survival in this type of patient, but we advise against its use during the current crisis $(4-6,33)$.

Cytokines as IL- 6 have been directly related to the most aggressive form of COVID-19. Hence, Immunotherapy with sipuleucel-T whose more frequently adverse events involve cytokine release, should not be given $(4,34)$.

Radium-223 must be further avoided. Although, it is associated with overall survival benefit by 3,6 months in patients with CRPC without visceral metastases compared to ADT alone, it is also associated to hematologic toxicity (anemia, thrombocytopenia and neutropenia) and monthly risk visits to hospital for intravenous administration (35).

As a result of the lack of benefit in overall survival with the administration of denosumab or zoledronic acid we propose to delay its introduction due to their potential toxicity (e.g., osteonecrosis of the jaw, hypocalcaemia) $(36,37)$. In those cases where treatment has been already started, denosumab can be maintained in its monthly subcutaneous administration while zoledronic acid, which requires monthly hospital intravenous administration, should be delayed (Table-4).

\section{DISCUSSION}

$\mathrm{PCa}$ is the second most common cancer in men worldwide (behind lung cancer) and the first one in Europe with a higher incidence in developed countries as a consequence of screening programs (38). Therefore, this pathology represents an important percentage of the burden of work carried out in Uro-Oncology units, being RP one of the most frequent operating room procedures performed by urologists with a rising trend during recent years (39).

While the management of PCa was already complex and under constant debate $(15,16)$, the current global pandemic has further complicated the treatment algorithm of this pathology. Additionally, all current recommendations are not based on robust evidence, but mostly expert consensus. In this sense, at most the PCa treatment recommendations in "EAU guidelines recommendations to the COVID 19 era" have level 2-3 evidence (6).

Elective definitive PCa treatments as RP as well as RT are being cancelled or postponed for an unknown time in view of the following points:

1. Patients suffering from cancer are at increased risk of infection and serious complications from COVID-19 (40).

2. Unknown SARS-CoV-2 infected patients who are asymptomatic and who have undergone a surgery are more likely to suffer from complications with a mortality rate of $20.5 \%$ (41).

3. Risk of SARS-CoV-2 infection in the surgical team as well as patients (iatrogenic exposure to the virus).

4. Need of hospital resources as PPE, Hospital/ICU beds and ventilators.

In regard to definitive treatment choice, we must keep in mind that replacing most of RP by RT could not be the universal solution. In a well-balanced scenario, both treatments coexist and resources should be adapted to needs. Supposing that all patients suitable to undergo active treatment are treated with RT, treatment waiting time would be dramatically increased resulting in treatment delay. The potential solution may create a new problem. Hence, we advise to considerer RP as a potential curative treatment during and after COVID 19 pandemic. In this sense, it has been shown that in localized low and intermediate-risk PCa patients, 6 to 9 months of delay from biopsy to RP is associated to an increased risk of BCR or clinical recurrence at 5 years lower than 18\% and $0.6 \%$, respectively. While in high risk patients the risk of BCR is higher (close to 24\% after 9-12 from the biopsy), short term ADT might protect them until surgery in 3-6 months (42). It is important to take into consideration that the studies which lead us to avoid ADT prior to RP due to a lack of survival benefit (neither a detriment) compared to immediate surgery are the same which support the 
use of neoadjuvant ADT in those patients whose surgery is forced to be delayed during COVID19 pandemic (20).

The special situation that urologists face today force all of us not only to think about when but also how we must treat our patients.

All elective visits should be postponed or transitioned to telehealth visits to further reduce exposure risk. For those patients who must be seen in clinic, social distancing should be promoted to ensure minimal contact with staffs and other patients (43).

Once SARS-CoV-2 was confirmed as global pandemic by WHO, and community transmission was accepted, all patients must be considered suspected cases until proven otherwise. Therefore, we consider that all patients should be tested prior to any surgery. In case it is not possible, telephone interview depicting symptomatic or oligo-symptomatic cases could be an alternative option ( 9 , 44). Additionally, in those case where abdominal tomography image is required, thorax imagine should be added at the same time.

Testing of elective patients is recommended within 48 hours prior to surgery. SARS-CoV-2 positive patients or clinically suspected patients should have their $\mathrm{CaP}$ intervention postponed as far as possible (6).

Several studies have proven transmission of different viruses during surgery $(45,46)$. According to a recent publication, this risk could be higher during laparoscopic procedures compared to open ones (47). This is due to the concentrated aerosol in the abdominal cavity formed during the operation being released suddenly when trocars are removed, small incisions are done or instruments are exchanged (48). In addition, airborne transmission is possible through intubation and extubation. This fact has led the EAU Robotic Urology Section to propose some recommendations to safeguard the health of the surgical staff (9).

However, we shouldn't forget that not all urological cancers are PCa. The extent of therapeutic alternatives for $\mathrm{PCa}$ in its different stages drive us to considerer all of them during the lack of medical and surgical resources in favour of non-deferrable treatments such as cystectomies, trans-urethral resection of high volume tumours, big mass nephrectomies, or orchiectomies.

\section{CONCLUSION}

As a consequence of COVID-19 pandemic several measures have been taken in order to reduce the fast spread of the virus, to protect health professionals from infection during their work, to guarantee the health of in-patients, and to ensure the availability of health resources to address the vast number of patients suffering from the coronavirus disease. Subsequently, clinical and surgical strategies in Urology have been forced to adapt to the changes brought about by COVID-19.

Since PCa prognosis is generally favorable, we can safely delay most of the biopsies while AS must almost be mandatory in those patients with low risk PCa. Furthermore, the existence of therapeutic alternatives such as ADT allows us to defer definitive local treatment in many cases of intermediate and high risk PCa, assuming a believably not too significant impact on oncological outcomes. In regard to metastatic castration resistant $\mathrm{PCa}$, combination therapies with novel drugs such as abiraterone, apalutamide, darolutamide or enzalutamide should be considered in high risk diseases whereas their secondary effects could be managed by telehealth. Chemotherapy or Radium-223 must be avoided because of haematological toxicity and frequent hospital visits. We advise against the use of Sipuleucel-T given the risk of cytokines reaction.

Nevertheless, each PCa case must be considered individually and the proposed recommendations should constantly adapt to the epidemiological evolution of the situation.

\section{ABBREVIATIONS}

$\mathrm{PCa}=$ Prostate Cancer

$\mathrm{CRPC}=$ Castration Resistant Prostate Cancer

$\mathrm{RT}=$ Radiotherapy

$\mathrm{RP}=$ Radical Prostatectomy

ADT $=$ Androgen Deprivation Therapy 


\section{CONFLICT OF INTEREST}

None declared.

\section{REFERENCES}

1. Gillessen S, Attard G, Beer TM, Beltran H, Bjartell A, Bossi $A$, et al. Management of Patients with Advanced Prostate Cancer: Report of the Advanced Prostate Cancer Consensus Conference 2019. Eur Urol. 2020; 77:508-47.

2. Lu R, Zhao X, Li J, Niu P, Yang B, Wu H, et al. Genomic characterisation and epidemiology of 2019 novel coronavirus: implications for virus origins and receptor binding. Lancet. 2020; 395:565-74.

3. [No authors]. WHO . Coronavirus disease 2019 ( COVID-19) : situation report, 46. World Health Organization. Available at. $<$ <ttps://apps.who.int/iris/handle/10665/331443>. Accessed May, 10, 2020

4. [No authors]. Care of Prostate Cancer During the Covid-19 pandemic: Recommendations of the NCCN. Management of Prostate Cancer During the COVID-19 Pandemic. Available at. https://www.nccn.org/covid-19/pdf/NCCN_PCa_COVID_ guidelines.pdf. Accessed May, 10, 2020

5. [No authors]. British Association of Urological Surgeons. COVID-19 strategy for the Interim management of Prostate Cancer Prepared by the BAUS Section of Oncology. 2020. Available at. https://caunet.org/wp-content/uploads/2020/04/BAUS-OncologyCOVID-19-Prostate.pdf. Accessed May, 10, 2020

6. Ribal MJ, Cornford P, Briganti A, Knoll T, Gravas S, Babjuk M, etal. European Association of Urology Guidelines Office Rapid Reaction Group: An Organisation-wide Collaborative Effort to Adapt the European Association of Urology Guidelines Recommendations to the Coronavirus Disease 2019 Era. Eur Urol. 2020: S0302-2838, 30324-9. Available at. <https:// uroweb.org/wp-content/uploads/EAU-Guidelines-OfficeRapid-Reaction-Group-An-organisation-wide-collaborativeeffort-to-adapt-the-EAU-guidelines-recommendations-tothe-COVID-19-era.pdf>. Accessed May, 10, 2020

7. [No authors]. Care of Prostate Cancer During the COVID-19 pandemic: Recommendations of the NCCN. Recommendations for Prostate Cancer Early Detection During the COVID-19 Pandemic 4/1/2020. Available at https://www.nccn.org/covid-19/pdf/Prostate_Early_ Detection.pdf. Accessed May, 10, 2020
8. Katz EG, Stensland KS, Mandeville JA, MacLachlan LS, Moinzadeh A, Sorcini A, et al. Triaging Office-Based UrologY Procedures During the COVID-19 Pandemic. J Urol. 2020. Epub ahead of print.

9. Mottrie A. ERUS (EAU Robotic Urology Section) guidelines during COVID-19 emergency.[Internet]. Available at. <https://uroweb.org/wp-content/uploads/ERUSguidelines-for-COVID-def.pdf>

10. Goldman HB, Haber GP. Recommendations for Tiered Stratification of Urological Surgery Urgency in the COVID-19 Era. J Urol. 2020: Epub ahead of print.

11. Zaorsky NG, Yu JB, McBride SM, Dess RT, Jackson WC, Mahal BA, et al. Prostate Cancer Radiotherapy Recommendations in Response to COVID-19. Adv Radiat Oncol. 2020. Epub ahead of print.

12. Tosoian JJ, Mamawala M, Epstein JI, Landis P, Macura KJ, Simopoulos DN, et al. Active Surveillance of Grade Group 1 Prostate Cancer: Long-term Outcomes from a Large Prospective Cohort. Eur Urol. 2020; 77:675-82.

13. Wilt TJ, Jones KM, Barry MJ, Andriole GL, Culkin D, Wheeler T, et al. Follow-up of Prostatectomy versus Observation for Early Prostate Cancer. N Engl J Med. 2017; 377:132-42.

14. Hamdy FC, Donovan JL, Lane JA, Mason M, Metcalfe C, Holding $\mathrm{P}$, et al. 10-Year Outcomes after Monitoring, Surgery, or Radiotherapy for Localized Prostate Cancer. N Engl J Med. 2016; 375:1415-24.

15. Mohler JL, Antonarakis ES, Armstrong AJ, D'Amico AV, Davis BJ, Dorff T, et al. Prostate Cancer, Version 2.2019, NCCN Clinical Practice Guidelines in Oncology. J Nat Compr Canc Netw. 2019; 17:479-505.

16. Mottet N, Cornford P, van den Bergh RCN, Briers E, De Santis M, Fanti F, et al. Prostate Cancer. EAU Guidelines Associates. [Internet]. Available at. <https://uroweb.org/ guideline/prostate-cancer/>.

17. Kumar S, Shelley M, Harrison C, Coles B, Wilt TJ, Mason MD. Neo-adjuvant and adjuvant hormone therapy for localised and locally advanced prostate cancer. Cochrane Database Syst Rev. 2006; (4):CD006019.

18. Pisansky TM, Hunt D, Gomella LG, Amin MB, Balogh $A G$, Chinn DM, et al. Duration of androgen suppression before radiotherapy for localized prostate cancer: radiation therapy oncology group randomized clinical trial 9910. J Clin Oncol. 2015; 33:332-9. 
19. Yamamoto T, Musunuru HB, Vesprini D, Zhang L, Ghanem G, Loblaw A, et al. Metastatic Prostate Cancer in Men Initially Treated with Active Surveillance. J Urol. 2016; 195:1409-14.

20. Bill-Axelson A, Holmberg L, Garmo H, Taari K, Busch C, Nordling S, et al. Radical Prostatectomy or Watchful Waiting in Prostate Cancer - 29-Year Follow-up. N Engl J Med. 2018; 379:2319-29.

21. McKay RR, Ye H, Xie W, Lis R, Calagua C, Zhang Z, et al. Evaluation of Intense Androgen Deprivation Before Prostatectomy: A Randomized Phase II Trial of Enzalutamide and Leuprolide With or Without Abiraterone. J Clin Oncol. 2019; 37:923-31.

22. Vale CL, Brihoum M, Chabaud S, Sargos P, Sydes MR, Tierney $\mathrm{JF}$, et al. Adjuvant or salvage radiotherapy for the treatment of localised prostate cancer? A prospectively planned aggregate data meta-analysis. Annals of Oncology 2019;30 (Suppl 5), v883. Available at. <https://www.annalsofoncology.org/article/ S0923-7534(19)60401-1/abstract>.

23. Van den Broeck T, van den Bergh RCN, Arfi N, Gross T, Moris $L$, Briers E, et al. Prognostic Value of Biochemical Recurrence Following Treatment with Curative Intent for Prostate Cancer: A Systematic Review. Eur Urol. 2019; 75:967-87.

24. Fizazi K, Tran N, Fein L, Matsubara N, Rodriguez-Antolin A, Alekseev BY, et al. Abiraterone plus Prednisone in Metastatic, Castration-Sensitive Prostate Cancer. N Engl J Med. 2017; 377:352-60.

25. Chi KN, Agarwal N, Bjartell A, Chung BH, Pereira de Santana Gomes AJ, Given R, Juárez Soto Á, et al. Apalutamide for Metastatic, Castration-Sensitive Prostate Cancer. N Engl J Med. 2019; 381:13-24.

26. Armstrong AJ, Szmulewitz RZ, Petrylak DP, Holzbeierlein J, Villers A, Azad A, et al. ARCHES: A Randomized, Phase III Study of Androgen Deprivation Therapy With Enzalutamide or Placebo in Men With Metastatic Hormone-Sensitive Prostate Cancer. J Clin Oncol. 2019; 37:2974-86.

27. Wang D, Hu B, Hu C, Zhu F, Liu X, Zhang J, et al. Clinical Characteristics of 138 Hospitalized Patients With 2019 Novel Coronavirus-Infected Pneumonia in Wuhan, China. JAMA. 2020; 323:1061-9.

28. Russell CD, Millar JE, Baillie JK. Clinical evidence does not support corticosteroid treatment for 2019-nCoV lung injury. Lancet. 2020; 395:473-5.

29. de Bono JS, Logothetis CJ, Molina A, Fizazi K, North S, Chu $L$, et al. Abiraterone and increased survival in metastatic prostate cancer. N Engl J Med. 2011; 364:1995-2005.
30. Ryan CJ, Smith MR, Fizazi K, Saad F, Mulders PF, Sternberg $\mathrm{CN}$, et al. Abiraterone acetate plus prednisone versus placebo plus prednisone in chemotherapy-naive men with metastatic castration-resistant prostate cancer (COU-AA-302): final overall survival analysis of a randomised, double-blind, placebo-controlled phase 3 study. Lancet Oncol. 2015; 16:152-60.

31. Scher HI, Fizazi K, Saad F, Taplin ME, Sternberg CN, Miller K, et al. Increased survival with enzalutamide in prostate cancer after chemotherapy. N Engl J Med. 2012; 367:1187-97.

32. Beer TM, Armstrong AJ, Rathkopf DE, Loriot Y, Sternberg CN, Higano CS, et al. Enzalutamide in metastatic prostate cancer before chemotherapy. N Engl J Med. 2014; 371:424-33.

33. Gillessen S, Powles T. Advice Regarding Systemic Therapy in Patients with Urological Cancers During the COVID-19 Pandemic. Eur Urol. 2020; 77:667-8.

34. Mulders PF, De Santis M, Powles T, Fizazi K. Targeted treatment of metastatic castration-resistant prostate cancer with sipuleucel-T immunotherapy. Cancer Immunol Immunother. 2015; 64:655-63.

35. Parker C, Nilsson S, Heinrich D, Helle SI, O'Sullivan JM, Fosså SD, et al. Alpha emitter radium-223 and survival in metastatic prostate cancer. N Engl J Med. 2013; 369:213-23.

36. Smith MR, Saad F, Coleman R, Shore N, Fizazi K, Tombal $B$, et al. Denosumab and bone-metastasis-free survival in men with castration-resistant prostate cancer: results of a phase 3, randomised, placebo-controlled trial. Lancet. 2012; 379:39-46.

37. Saad F, Gleason DM, Murray R, Tchekmedyian S, Venner $P$, Lacombe $L$, et al. A randomized, placebo-controlled trial of zoledronic acid in patients with hormone-refractory metastatic prostate carcinoma. J Natl Cancer Inst. 2002; 94:1458-68.

38. Ferlay J, Ervik M, Lam F, Colombet M, Mery L, Piñeros M, et al. Cancer Today (powered by GLOBOCAN 2018). IARC. [Internet]. Available at. < https://publications.iarc.fr/ Databases/larc-Cancerbases/Cancer-Today-Powered-ByGLOBOCAN-2018--2018>.

39. van den Bergh R, Gandaglia G, Tilki D, Borgmann $H$, Ost P, Surcel C, et al. Trends in Radical Prostatectomy Risk Group Distribution in a European Multicenter Analysis of 28572 Patients: Towards Tailored Treatment. Eur Urol Focus. 2019; 5:171-8.

40. Liang W, Guan W, Chen R, Wang W, Li J, Xu K, et al. Cancer patients in SARS-CoV-2 infection: a nationwide analysis in China. Lancet Oncol. 2020; 21:335-7. 
41. Lei S, Jiang F, Su W, Chen C, Chen J, Mei W, et al. Clinical characteristics and outcomes of patients undergoing surgeries during the incubation period of COVID-19 infection. EClinicalMedicine. 2020. Epub ahead of print.

42. Fossati N, Rossi MS, Cucchiara V, Gandaglia G, Dell'Oglio P, Moschini M, et al. Evaluating the effect of time from prostate cancer diagnosis to radical prostatectomy on cancer control: Can surgery be postponed safely? Urol Oncol. 2017; 35:150. e9-150.e15.

43. Connor MJ, Winkler M, Miah S. COVID-19 pandemic - is virtual urology clinic the answer to keeping the cancer pathway moving? BJU Int. 2020;125:E3-4.

44. Simonato A, Giannarini G, Abrate A, Bartoletti R, Crestani $A$, De Nunzio $C$, et al. Pathways for urology patients during the COVID-19 pandemic. Minerva Urol Nefrol. 2020. Epub ahead of print.

45. Gloster HM Jr, Roenigk RK. Risk of acquiring human papillomavirus from the plume produced by the carbon dioxide laser in the treatment of warts. J Am Acad Dermatol. 1995; 32:436-41.
46. Johnson GK, Robinson WS. Human immunodeficiency virus-1 (HIV-1) in the vapors of surgical power instruments. J Med Virol. 1991; 33:47-50.

47. Li Cl, Pai JY, Chen CH. Characterization of smoke generated during the use of surgical knife in laparotomy surgeries. J Air Waste Manag Assoc. 2020; 70:324-32.

48. Zheng $\mathrm{MH}$, Boni L, Fingerhut A. Minimally Invasive Surgery and the Novel Coronavirus Outbreak: Lessons Learned in China and Italy. Ann Surg. 2020. Epub ahead of print.
Correspondence address:

Lara Rodriguez Sanchez, MD Department of Urology, Institut Mutualiste Montsouris, Université Paris-Descartes, Paris, France Telephone: +34 615 192-356 E-mail: rodriguezsanchezlara@gmail.com 